\title{
Efficacy of a Synergistic Blend of Organic Acids and B-1,4 Mannobiose on Cecal Salmonella Counts and Growth Performance in Salmonella Challenged Broiler Chickens: A Meta-Analysis
}

\author{
Sandra J. A. van Kuijk *(D) and Yanming Han * \\ Trouw Nutrition R\&D, Stationsstraat 77, 3811 MH Amersfoort, The Netherlands \\ * Correspondence: Sandra.van.kuijk@trouwnutrition.com (S.J.A.v.K.); \\ Yanming.Han@trouwnutrition.com (Y.H.); Tel.: +31-6-820-484-84 (S.J.A.v.K.)
}

Citation: van Kuijk, S.J.A.; Han, Y. Efficacy of a Synergistic Blend of Organic Acids and B-1,4 Mannobiose on Cecal Salmonella Counts and Growth Performance in Salmonella Challenged Broiler Chickens: A Meta-Analysis. Animals 2021, 11, 2988. https://doi.org/10.3390/ani11102988

Academic Editor: Palmy Jesudhasan

Received: 2 September 2021

Accepted: 13 October 2021

Published: 17 October 2021

Publisher's Note: MDPI stays neutral with regard to jurisdictional claims in published maps and institutional affiliations.

Copyright: () 2021 by the authors. Licensee MDPI, Basel, Switzerland. This article is an open access article distributed under the terms and conditions of the Creative Commons Attribution (CC BY) license (https:// creativecommons.org/licenses/by/ $4.0 /)$.
Simple Summary: Salmonella may cause serious diarrhea in consumers of poultry products. Control of Salmonella in poultry with antibiotics has caused antimicrobial resistance issues. Salmonella can colonize the gut of chickens, meaning that an in-feed solution may prevent infection. A synergistic blend containing organic acids and B-1,4 mannobiose as main ingredients were developed to reach different parts of the gut. It was hypothesized that this synergistic blend decreases Salmonella contamination of chickens. Several, non-published, studies have been performed to test the effect of this synergistic blend in chickens infected with Salmonella. The results of these studies were combined into a large meta-analysis to draw conclusions regardless of study design and geographical location. This state-of-the-art statistical method did show that feeding the synergistic blend to chickens could decrease Salmonella in comparison to a control diet. This decrease was most clear during the first 14 days after initiation of the Salmonella infection. In addition, the birds did grow more efficiently when the synergistic blend was fed.

Abstract: This study aimed at investigating the effect of a functional synergistic feed additive blend, containing organic acids and $\$-1,4$ mannobiose, on cecal Salmonella counts and growth performance of broiler chickens. A meta-analysis combining 13 individual studies, executed in Salmonella-challenged broilers comparing a control diet with and without the synergistic blend, was performed. Cecal Salmonella colonies and overall growth performance were measured. Raw data from all studies were combined and analyzed using PROC MIXED in SAS, taking the within and between-study variation into account. In the first 14 days post-inoculation (DPI), cecal Salmonella was reduced by $0.429 \log \mathrm{CFU} / \mathrm{g}$ ( $p=0.011, n=10$ comparisons from five studies) when feeding the synergistic blend compared to the control group. During 15-34 DPI, the overall means were not different between treatments ( $0.069 \log \mathrm{CFU} / \mathrm{g} ; p=0.519, n=12$ comparisons from eight studies). The feed conversion ratio was improved when feeding the synergistic blend compared to the control diet (1.474 vs. 1.482, respectively; $p=0.002$ ). In conclusion, feeding a synergistic blend, containing organic acids and $\beta-1,4$ mannobiose amongst other ingredients, reduced cecal Salmonella counts during the first 14 DPI and improved growth performance of Salmonella challenged broilers compared to a control diet.

Keywords: broiler chicken; growth performance; mannobiose; meta-analysis; organic acids; Salmonella

\section{Introduction}

Salmonella is a major foodborne pathogen that not only results in direct food safety issues by causing diarrhea and other health problems in humans; indirectly, potentially more severe problems can occur, as several Salmonella serovars are described to be resistant to one or more antibiotics. In a recent overview [1], the median prevalence values of Salmonella in broiler chickens, raw chicken meat, and in eggs and egg-laying hens were 
$40.5 \%, 30 \%$, and $40 \%$, respectively. Antimicrobial therapy is the first choice of treatment for this bacterial infection; however, antimicrobial resistance has become a problem due to the misuse of antibiotics, both in human medicine and animal production [1]. In the period from 2000-2004, 15\% of the clinical Salmonella isolates in Europe were identified as multi-drug resistant, and this number was even up to $84 \%$ of the clinical isolates in the USA in 2005-2006 [2]. More recently, published in 2020 summarizing 45 scientific publications showing an incidence of multi-drug resistant Salmonella in $91.1 \%$ of the poultry farms across the world [1]. Between 15 and 35\% of the Salmonella related foodborne illnesses are related to poultry meat Hoffman et al. [3]. The spread of Salmonella in the poultry production chain is multifactorial and linked to feed, drinking water, the hatchery, pests, wild birds, insects, infected dead or alive birds, litter, personnel, equipment and vehicles [4-8]. Since feed can be a significant source for Salmonella contamination [4], an in-feed solution is postulated as one of the key steps in mitigating Salmonella contamination. On top of other measures, in-feed solutions focusing on strengthening natural barriers in the intestinal tract can be effective in reducing the infection and prevalence of Salmonella on farms.

Several in-feed solutions have been described in the literature. Infection can be prevented via lowering the $\mathrm{pH}$ of the gastrointestinal tract, for example via organic acids, specifically (encapsulated) short chain fatty acids (SCFA) [9-11]. Whereas SCFA act mostly in the upper gastrointestinal tract, medium chain fatty acids (MCFA) having a greater antibacterial activity against Salmonella are released later in the gastrointestinal tract [5]. However, to reach the lowest parts of the gastrointestinal tract, MCFA need encapsulation $[5,10,12]$. The coated form of butyric acid has been described to be effective in Salmonella control in the cecum of broiler chickens challenged with Salmonella Enteritidis, indicating that the coating of butyrate results in effects in the lower gastrointestinal tract [12]. Sodium butyrate was shown to improve the villi and mucosa of the intestines and in general has a beneficial effect on gut microbiota [13], general gut health and growth performance when compared to a negative control or antibiotic growth promotors [14]. Hydrolyzed copra meal is an ingredient containing significant amounts of $B-1,4$ mannobiose, a compound described to have an effect on Salmonella by increasing phagocytosis by macrophages in vitro [15] and reducing Salmonella in the cecum of challenged broiler chickens in vivo [16]. Because of the multifactorial nature of Salmonella infections and differences in modes of action of the aforementioned ingredients, a blend of different feed ingredients may have synergistic effects in targeting Salmonella. In addition, the complex nature of Salmonella infections often causes variable results in scientific experiments, especially when these are done under different circumstances. The objective of the present study was to investigate the overall effect of a synergistic feed additive blend including B-1,4 mannobiose, SCFA's, MCFA's, and microencapsulated butyrate on Salmonella counts in the cecum of Salmonella challenged broiler chickens, regardless of experimental conditions. Therefore, several experiments performed under different circumstances were combined into one meta-analysis. It is hypothesized that fewer Salmonella would colonize the cecum in birds fed the synergistic blend compared to the control birds. In addition, it is hypothesized that the synergistic concept not only affects Salmonella counts in the cecum but also improves the growth performance of Salmonella-challenged broiler chickens.

\section{Materials and Methods}

All animal procedures in the different studies were approved by the local animal committees according to the standard procedures of the universities/institutes, where the studies were performed.

\subsection{Selection of Studies}

The following inclusion criteria were maintained: (1) studies must have been done in broiler chickens starting from day-old; (2) broiler chickens must have been challenged at any age with any serovar of Salmonella except for pathogenic serovars for poultry; (3) Salmonella-challenged birds fed the synergistic blend must be compared to Salmonella- 
challenged birds fed a non-supplemented control diet; (4) the raw data must be available for the analysis. Studies were excluded from the meta-analysis when: (1) the inclusion criteria were not fulfilled; (2) data of the study were regarded as unreliable due to (unforeseen) circumstances; (3) Salmonella counts were lower than 3 log CFU/g cecal digesta during the first 20 days of the study indicating a failed challenge model. All considered studies were performed at Trouw Nutrition, or with collaborations of Trouw Nutrition. None of the studies were published.

\subsection{Study Design}

All studies were done with broiler chickens starting from one day old. In all studies, birds received a Salmonella challenge but the age at initiation of the challenge differed per study. The age at introduction of Salmonella challenge varied between day 1 and 10 and is summarized in Table 1 for each individual study. The serovars used in the different studies are summarized in Table 1, all were isolated from the field. Two types of challenges were used (Table 1): (1) An infection model, where all birds in a pen were inoculated with the same amount of inoculum; (2) A seeder model, where a selected number of birds per pen were inoculated with the respective Salmonella serovar and placed in the pen to spread the infection to other birds. In all cases, the dietary treatments started on day 1 of the study, which was well before the Salmonella challenge was introduced. The dietary treatments consisted of either a control diet, formulated to meet or exceed the requirements set by National Research Council [17] following local practices, or the control diet including the synergistic blend (Fysal Fit-4, Trouw Nutrition, the Netherlands). The same blend was used in all individual studies and was a proprietary mixture of SCFA, MCFA, hydrolyzed copra meal (containing 13\% B-1,4 mannobiose) and microencapsulated sodium salts of butyric acid. The synergistic blend was included at $3 \mathrm{~kg} / \mathrm{t}$ in the first feeding phase of all studies, up to a maximum of 23 days of the study, and then lowered to a minimum of $1 \mathrm{~kg} / \mathrm{t}$ until the end of each study.

Table 1. Studies included in the meta-analysis.

\begin{tabular}{|c|c|c|c|c|c|c|c|}
\hline Study Number & Facility & Country & $\begin{array}{l}\text { Study Duration } \\
\text { (Days) }\end{array}$ & Model & $\begin{array}{l}\text { Salmonella } \\
\text { Serovar }\end{array}$ & $\begin{array}{c}\text { Day of } \\
\text { Inoculation }\end{array}$ & Included Parameters ${ }^{1}$ \\
\hline 01 & $\begin{array}{l}\text { Trouw Nutrition } \\
\text { Poultry Research Centre }\end{array}$ & Spain & 32 & Infection & Enteritidis & 7 & $\begin{array}{c}\text { Growth performance, } \\
15-34 \text { DPI }\end{array}$ \\
\hline 02 & $\begin{array}{l}\text { Trouw Nutrition } \\
\text { Poultry Research Centre }\end{array}$ & Spain & 42 & Infection & Typhimurium & 8 & $\begin{array}{c}\text { Growth performance, } \\
15-34 \text { DPI }\end{array}$ \\
\hline 03 & $\begin{array}{l}\text { Trouw Nutrition } \\
\text { Poultry Research Centre }\end{array}$ & Spain & 35 & Infection & Enteritidis & 8 & $\begin{array}{l}\text { Growth performance, } \\
0-14 \text { DPI, 15-34 DPI }\end{array}$ \\
\hline 04 & $\begin{array}{c}\text { Trouw Nutrition } \\
\text { Poultry Research Centre }\end{array}$ & Spain & 42 & Seeder & Enteritidis & 8 & Growth performance \\
\hline 05 & $\begin{array}{l}\text { Trouw Nutrition } \\
\text { Poultry Research Centre }\end{array}$ & Spain & 32 & Infection & Enteritidis & 7 & $\begin{array}{l}\text { Growth performance, } \\
0-14 \text { DPI, 15-34 DPI }\end{array}$ \\
\hline 06 & $\begin{array}{l}\text { Trouw Nutrition } \\
\text { Poultry Research Centre }\end{array}$ & Spain & 39 & Seeder & Enteritidis & 7 & Growth performance \\
\hline 07 & $\begin{array}{l}\text { Trouw Nutrition } \\
\text { Poultry Research Centre }\end{array}$ & Spain & 42 & Infection & Enteritidis & 8 & $\begin{array}{l}\text { Growth performance, } \\
0-14 \text { DPI, 15-34 DPI }\end{array}$ \\
\hline 08 & $\begin{array}{l}\text { Trouw Nutrition } \\
\text { Poultry Research Centre }\end{array}$ & Spain & 33 & Infection & Enteritidis & 7 & $\begin{array}{c}\text { Growth performance, } \\
15-34 \text { DPI }\end{array}$ \\
\hline 09 & $\begin{array}{l}\text { Trouw Nutrition } \\
\text { Poultry Research Centre }\end{array}$ & Spain & 33 & Infection & Typhimurium & 7 & $\begin{array}{c}\text { Growth performance, } \\
15-34 \text { DPI }\end{array}$ \\
\hline 10 & Mercolab & Brazil & 35 & Seeder & Heidelberg & 3 & 0-14 DPI, 15-34 DPI \\
\hline 11 & USP & Brazil & 42 & Infection & Heidelberg & 10 & Growth performance \\
\hline 12 & IRTA & Spain & 35 & Seeder & Enteritidis & 1 & Growth performance \\
\hline 13 & Chulalongkorn & Thailand & 35 & Infection & Typhimurium & 7 & $\begin{array}{c}\text { Growth performance, } \\
0-14 \text { DPI }\end{array}$ \\
\hline
\end{tabular}

${ }^{1}$ Performance $=$ growth performance, $0-14$ DPI = Salmonella counts between days 0 and 14 post-inoculation, 15-34 DPI = Salmonella counts between days 15 and 35 post-inoculation.

\subsection{Parameters}

The meta-analysis included two major parts, one being the Salmonella data and the other one the growth performance for the overall study period. For the Salmonella assessment, a minimum of one, up to 10 birds per pen, dependent on the study design, were selected for the sampling of cecal digesta at different days post-inoculation. In studies 
$1,5,6,8$ and 9 , the complete content of one cecum per bird was used, while in the other studies $(3,4,7,10,11,12,13)$ the complete content of both ceca was used for Salmonella enumeration. In the case of a seeder model, seeder birds were excluded from the analysis. Salmonella counts were based on plating methods using Brilliant Green Agar with supplements (origin unknown) incubated at $37^{\circ} \mathrm{C}$ for $24 \mathrm{~h}$ (studies $01,03,05,07,08,09$ and 10 in Table 1), Bismuth Silfite Agar with supplemented Novobiocin and Salmonella growth supplement (origin unknown) incubated at $37^{\circ} \mathrm{C}$ for $24 \mathrm{~h}$ (study 02 in Table 1), or xylose lysine deoxycholate (XLD) agar containing nalidixic acid at $50 \mu \mathrm{g} / \mathrm{mL}$ (origin unknown) incubated at $42{ }^{\circ} \mathrm{C}$ for $24 \mathrm{~h}$ (study 13 on Table 1). Salmonella counts below the detection limit were regarded as negative (given a value of 0). Salmonella counts, as average per pen, were expressed as log colony forming units (CFU)/g cecal digesta.

Bodyweight was measured per pen at the start and end of each study. The feed intake was measured per pen for the overall study period. Growth performance was calculated for the total study period as average daily gain (ADG) in $\mathrm{g} /$ bird/day, average daily feed intake (ADFI) in g/bird/day and feed:gain ratio as feed conversion ratio (FCR) in g:g.

\subsection{Statistics}

All raw data were combined into one Excel file. Individual pens in each study were considered as the experimental unit. Statistical analysis was performed using the SAS software, version 9.4 (SAS Institute Inc., Cary, NC, USA). All parameters were compared between infected birds fed the synergistic blend and infected birds fed a control diet. Salmonella counts were split into two different data sets, one including counts between 0and 14-days post-inoculation (DPI) and another including counts between 15 and 34 DPI. All parameters were analyzed using the MIXED procedure in SAS. In each model the within-study variation and between-study variation were included in the random and repeated statement, respectively, not assuming equal variances of treatments. Results were regarded as significant at $p<0.05$.

\section{Results}

\subsection{Selection of Studies}

Based on the inclusion and exclusion criteria as defined in the materials and methods, 13 out of 16 studies were selected for the meta-analysis. The first study excluded was because Salmonella Pullorum was used in the challenge model. The second study excluded was because the minimum infection level of $3 \log$ CFU/g Salmonella in the cecum was not reached within the first 20 days of the study. The third study excluded showed results that cannot be explained by any biological processes. In this latter study, instead of an expected steady decline of Salmonella counts, similar to the natural course of a Salmonella infection, a sudden sharp increase in Salmonella counts was observed. The reason for the unexpected observation in this study is unknown, therefore this study was removed from the meta-analysis.

Due to differences in the study set-ups, not all 13 selected studies could be included in the analysis for all parameters. Differences in timings of measurements, measurement methods (quantitative vs. qualitative Salmonella identification) and availability of growth performance data resulted in a different number of studies included for the individual parameters. Table 1 shows the included studies with their key characteristics and parameters.

\subsection{Cecal Salmonella Counts}

Figure 1 shows an overview of the cecal counts for the different DPI in all studies. It shows that the peak in Salmonella counts in the cecum appeared to happen during the first 14 DPI. For this reason, the meta-analysis has been grouped based on DPI with the first group being 0-14 DPI and the second from 15-34 DPI.

Five studies could be included in the analysis 0-14 DPI, due to the study designs. Figure 2 and Table 2 show the results of the meta-analysis 0-14 DPI. Figure 2 shows the mean difference in log CFU Salmonella counts in the cecum per study per DPI. The 
difference between the overall means of all studies is $0.429 \log$ CFU $/ g(p=0.011, n=10$ comparisons from five studies) in favor of the synergistic blend.

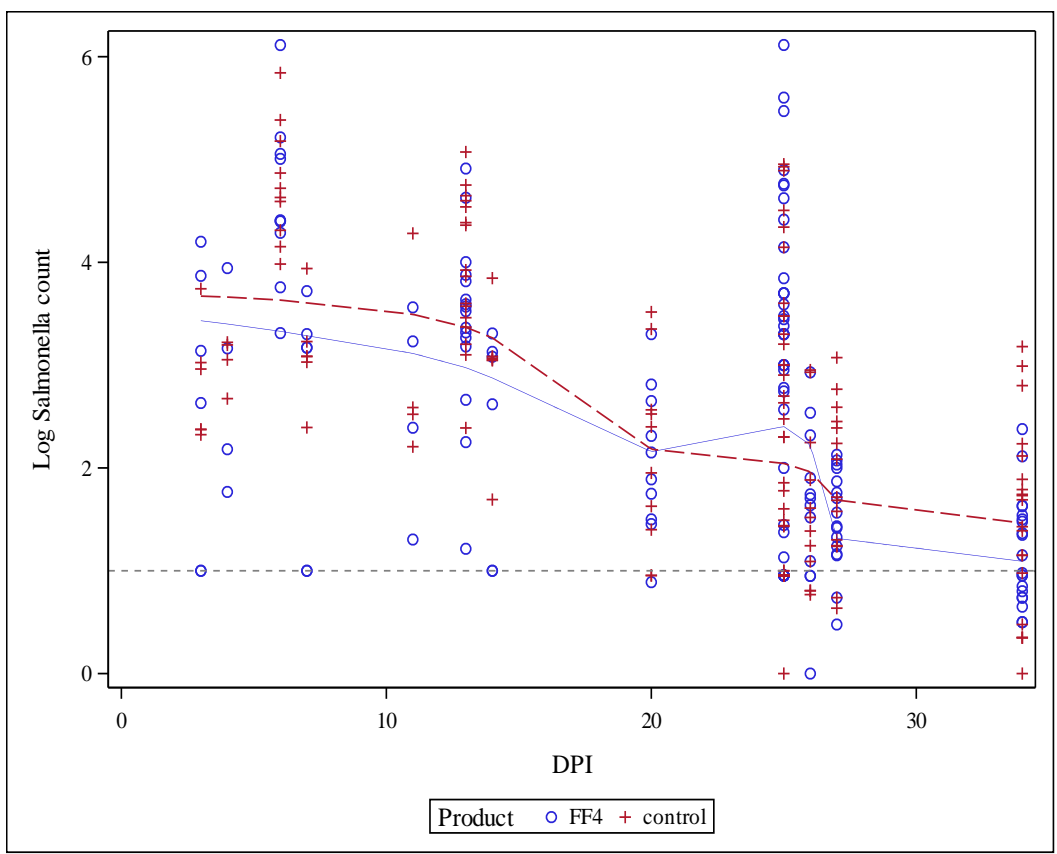

Figure 1. Individual Salmonella counts (log CFU/g) on different days post-inoculation (DPI). Each plus or circle represents one measurement per pen of the control or synergistic blend group respectively. The solid blue line is fitted through the data of the synergistic blend group, the dotted red line is fitted through the data of the control group. The vertical line represents where the split was made in the data set at 14 DPI.

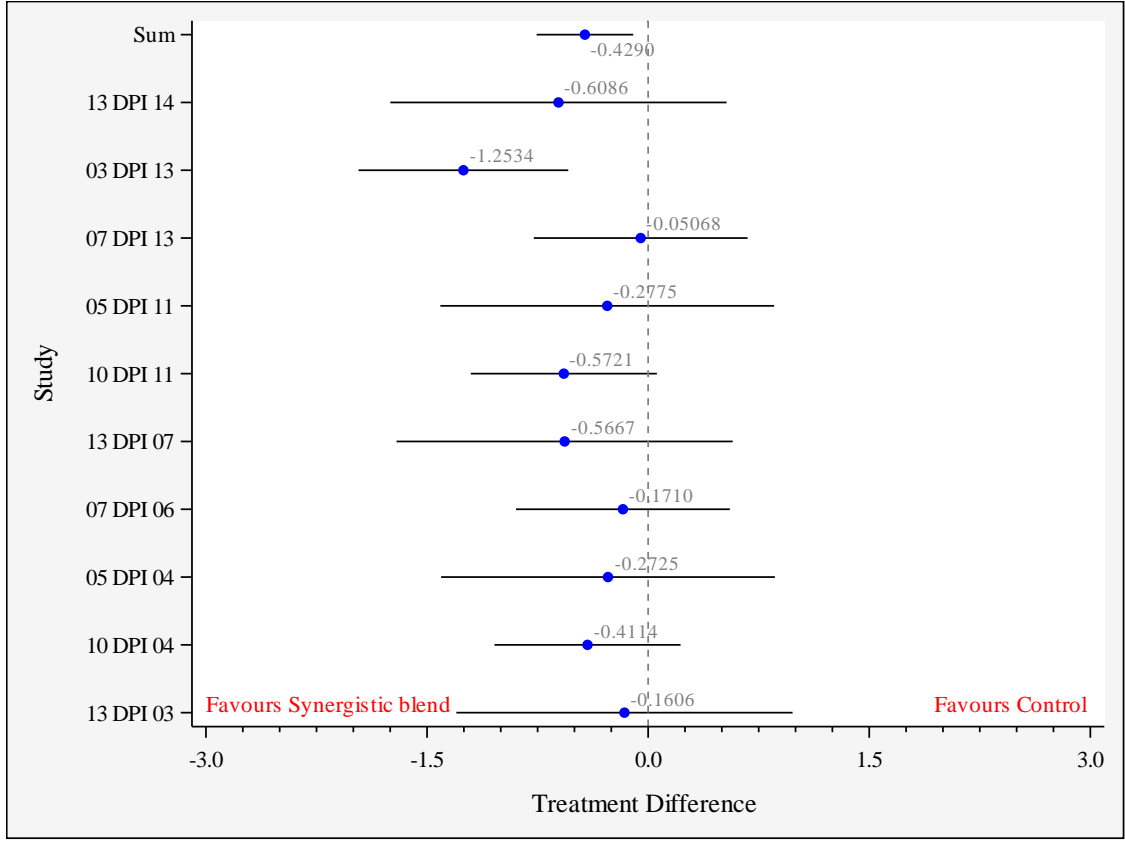

Figure 2. Forest plot showing the difference of the mean between control and synergistic blend (Treatment) for each study, and all studies combined (sum; $p=0.011$ ), $0-14$ days post-inoculation (DPI). The circles represent the log CFU/g difference between treatments, the bars represent the $95 \%$ confidence limits and the vertical line at 0 represents the value of no difference between treatments. Legend: XX DPI YY with XX = study number (see Table 1) and YY= days post-inoculation. 
Table 2. Meta-analysis of cecal Salmonella counts.

\begin{tabular}{|c|c|c|}
\hline Outcome & $\begin{array}{c}\text { Salmonella } \\
\text { 0-14 DPI } 1 \\
\text { (Log CFU/g) }\end{array}$ & $\begin{array}{l}\text { Salmonella } \\
\text { 15-34 DPI } \\
\text { (Log CFU/g) }\end{array}$ \\
\hline Mean-Control & $3.097^{\mathrm{a}}$ & 1.605 \\
\hline Mean-Synergistic blend & $2.669^{b}$ & 1.536 \\
\hline Difference of the means & -0.429 & -0.069 \\
\hline $\begin{array}{c}\text { Standard error of the } \\
\text { difference }\end{array}$ & 0.164 & 0.107 \\
\hline Lower $95 \%$ confidence limit & -0.757 & -0.281 \\
\hline Upper $95 \%$ confidence limit & -0.101 & 0.142 \\
\hline$p$-value & 0.011 & 0.519 \\
\hline
\end{tabular}

${ }^{1} 0-14$ DPI = Salmonella counts between days 0 and 14 post-inoculation, ${ }^{2}$ 15-34 DPI = Salmonella counts between days 15 and 35 post-inoculation. ${ }^{a, b}$ different superscripts within a column indicate significant difference between means $(p \leq 0.05)$.

Eight studies were included in the analysis of cecal Salmonella counts for 15-34 DPI. The results of this analysis are summarized in Figure 3 and Table 2. The overall means were not significantly different $(0.069 \log \mathrm{CFU} / \mathrm{g} ; p=0.519, n=12$ comparisons from eight studies) between the treatments.

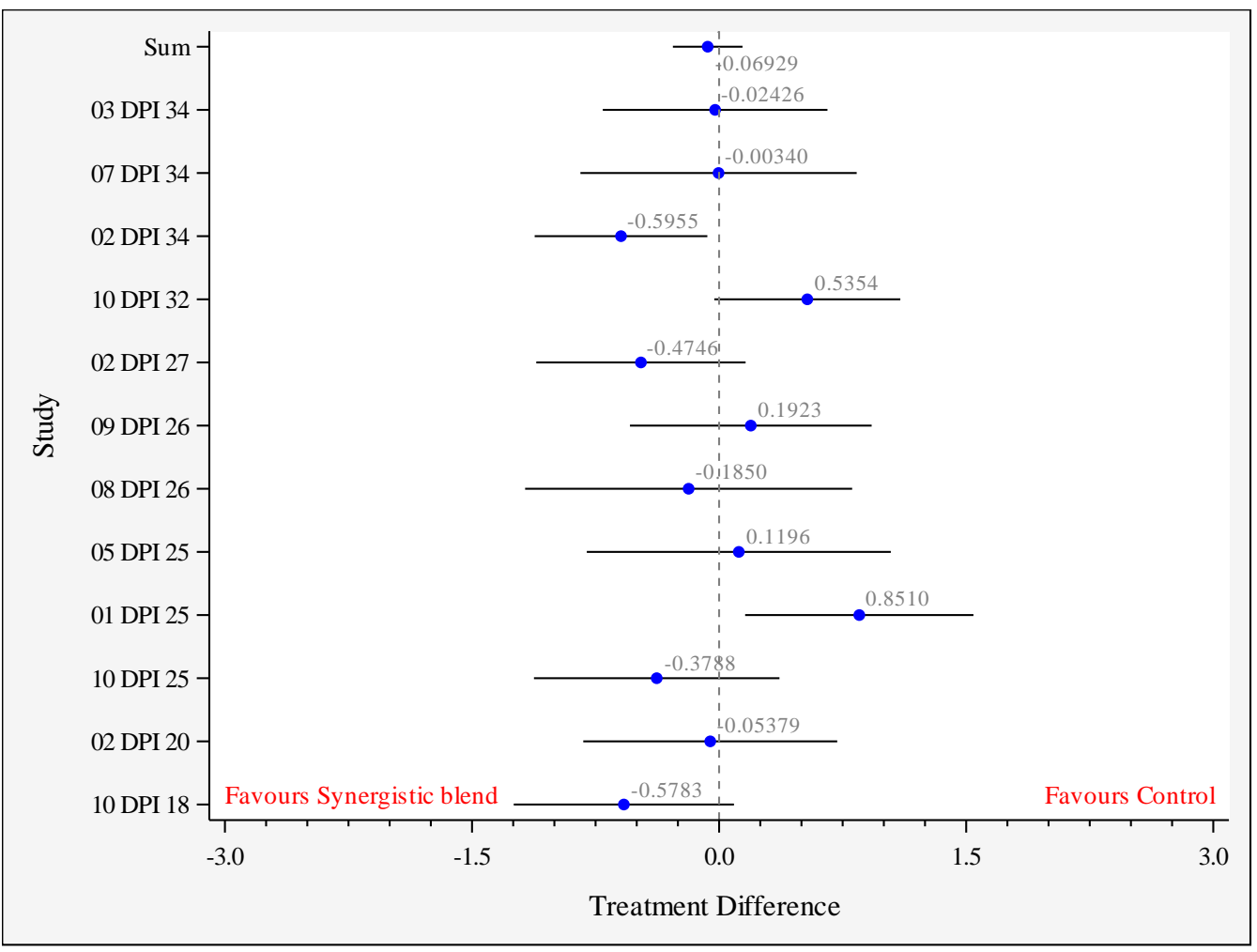

Figure 3. Forest plot showing the difference of the mean between control and synergistic blend (Treatment) for each study, and all studies combined (sum; $p=0.591$ ), between 15-34 days postinoculation (DPI). The circles represent the log CFU/g difference between treatments, the bars represent the $95 \%$ confidence limits and the vertical line at 0 represents the value of no difference between treatments. Legend: XX DPI YY with XX = study number (see Table 1) and YY= days post-inoculation.

\subsection{Growth Performance}

The growth performance results are summarized in Table 3. When including all studies as indicated in Table 1, there were no significant differences in final body weight, ADG and ADFI. The feed conversion ratio was significantly improved in the birds fed the 
synergistic blend compared to the control diet ( 1.474 vs. 1.482 respectively; $p=0.002, n=12$ comparisons, Figure 4).

Table 3. Results of the meta-analysis on growth performance for the overall study period.

\begin{tabular}{ccccc}
\hline Outcome & Final BW $^{\mathbf{1}} \mathbf{( g )}$ & ADG $^{\mathbf{2}}$ (g/Bird/Day) & ADFI $^{\mathbf{3}}$ (g/Bird/Day) & FCR (g:g) $^{\mathbf{4}}$ \\
\hline Mean-Control & 2304.5 & 62.31 & 92.36 & $1.482^{\mathrm{a}}$ \\
Mean-Synergistic blend & 2304.8 & 62.50 & 91.97 & $1.4744^{\mathrm{b}}$ \\
Difference between means & 0.28 & 0.188 & -0.386 & -0.0077 \\
Standard error of the difference & 18.64 & 0.291 & 0.396 & 0.0023 \\
Lower 95\% confidence limit & -36.45 & -0.388 & -1.179 & -0.0124 \\
Upper 95\% confidence limit & 37.01 & 0.764 & 0.407 & -0.0030 \\
p-value & 0.988 & 0.518 & 0.334 & 0.002 \\
\hline
\end{tabular}

${ }^{1} \mathrm{BW}=$ body weight, ${ }^{2}$ ADG $=$ average daily gain, ${ }^{3}$ ADFI $=$ average daily feed intake, ${ }^{4}$ FCR $=$ feed conversion ratio, ${ }^{a, b}$ different superscripts within a column indicate a significant difference between means $(p \leq 0.05)$.

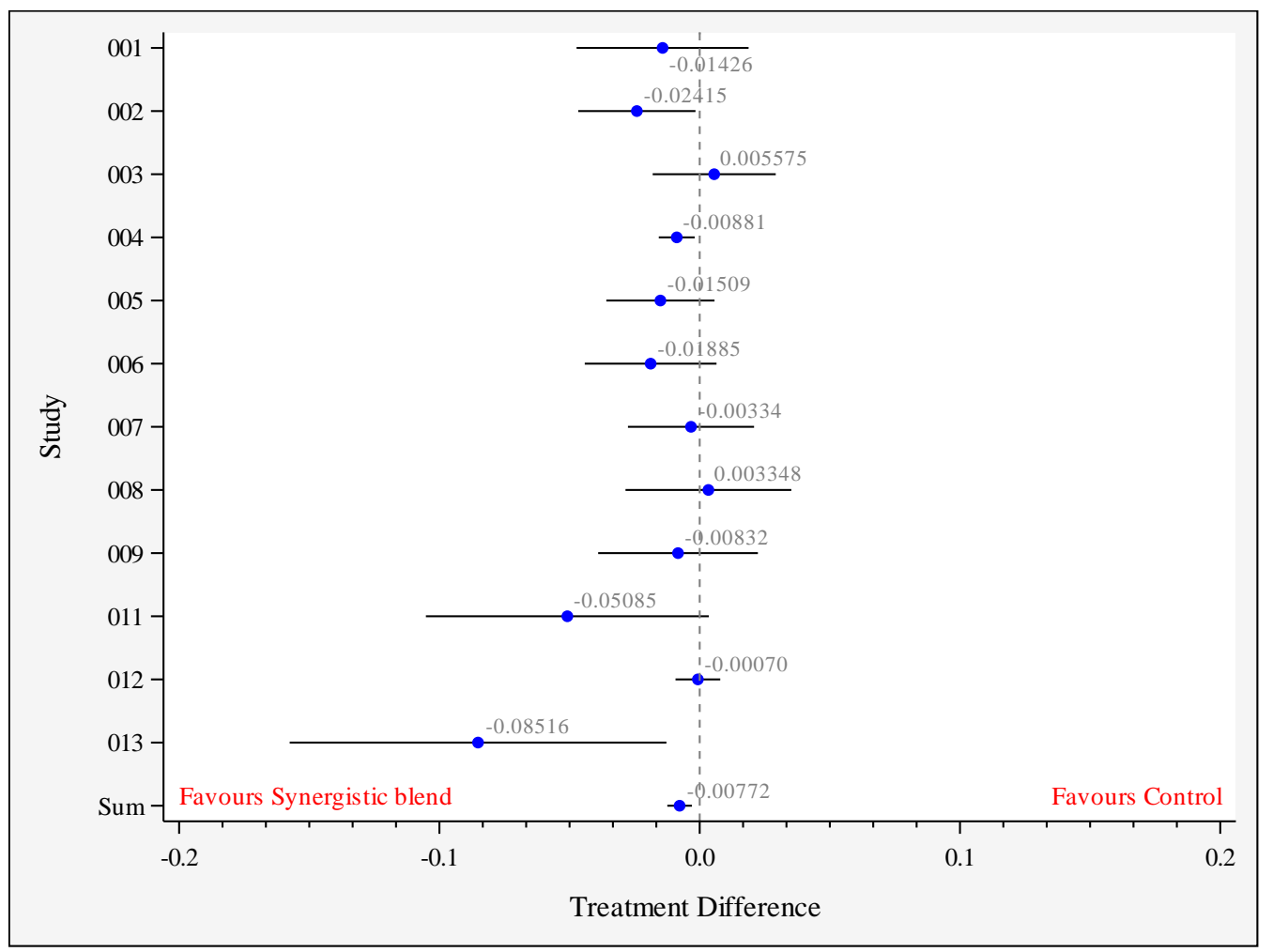

Figure 4. Forest plot for a feed conversion ratio of the overall study period between control and synergistic blend (Treatment). The circles represent the $\log \mathrm{CFU} / \mathrm{g}$ difference between treatments, the bars represent the $95 \%$ confidence limits and the vertical line at 0 represents the value of no difference between treatments. Legend: 0XX with XX = study number (See Table 1).

In line with the Salmonella count analysis, where not all studies could be included in each analysis, an additional analysis for growth performance was performed. Taking into account only the same five studies (study number 3, 5, 7, 10,13) used for the analysis on the Salmonella counts 0-14 DPI, a tendency towards an increased body weight of $21.8 \mathrm{~g}$ compared to the control diet (2317.7 g vs. $2295.0 \mathrm{~g} ; p=0.097, n=5$ comparisons) was observed for the chickens fed the synergistic feed additive blend, while the beneficial effect on FCR was not observed here.

\section{Discussion}

In total 13 studies were selected for inclusion in the meta-analysis. One study was excluded because of the use of Salmonella Pullorum. This serovar is known to be pathogenic for broiler chickens $[6,18]$. Salmonella Pullorum does cause disease in poultry, while the other serovars used in the current study will not cause any clinical signs in infected 
birds [18]. The pathogenicity of Salmonella Pullorum might influence infection and growth performance differently, compared to other serovars. Another study was excluded because it did not reach the minimum of $3 \log \mathrm{CFU} / \mathrm{g}$ cecal Salmonella counts. As the Salmonella detection methods used here have a detection limit between 1 and $2 \log \mathrm{CFU} / \mathrm{g}$, it is expected that any small reduction in birds having cecal counts below $3 \log \mathrm{CFU} / \mathrm{g}$ would result in undetectable or highly variable results.

All data combined as shown in Figure 1 indicated a peak in cecal Salmonella counts in the first 14 days post-inoculation, which is similar to the trends described by Stern [19] for birds at 4 days of age infected with three different Salmonella serovars. This decrease in Salmonella counts over time was attributed to the maturation of the intestinal flora of young chicks [19]. In the current study, the most significant differences were observed in the first 14 days post-inoculation. The lack of difference in data thereafter is likely related to an adaptive decrease in Salmonella colonization in growing chicks [19]. The current results confirm the natural course of Salmonella infection and show that interventions should be applied within the first 2 weeks when inoculation occurs at a younger age.

The serovars used in the current meta-analysis, Salmonella Enteritidis, Salmonella Typhimurium or Salmonella Heidelberg, represent the most common ones in Europe and the USA $[5,7,20,21]$. Not only is a food safety risk associated with these serovars, some, such as Salmonella Typhimurium, are described to have antibiotic resistance [2,22]. In commercial practice, more different serovars are being detected, such as Salmonella Infantis that is often related to antimicrobial resistance [8]. In this study, not enough data was available to draw specific conclusions per serovar. Therefore, future research should also look at the effect of different Salmonella control measures on each different serovar and more serovars should be studied. Next to different serovars, the studies included were different in regard to the challenge models as well as regional differences in animals and diets. None of these differences were considered in the statistical analysis due to insufficient data. However, the way the current analysis was executed indicates that the synergistic blend significantly reduces cecal Salmonella counts regardless of the study conditions.

The synergistic blend used in the current study is a mixture of active ingredients including SCFA, MCFA, hydrolyzed copra meal, sodium salts of butyric acid. The observed beneficial effects on Salmonella counts and growth performance cannot be attributed to specific single ingredients but is most likely the combination of the different modes of action of these ingredients. SCFA and MCFA are described to be effective against Salmonella $[5,9,23]$. Although these compounds may have an effect on Salmonella in feed already, or have a slight effect on the crop $\mathrm{pH}$, the most effect is to be expected if they manage to reach the lower part of the digestive tract, as this is the main colonization site of Salmonella [23]. In addition, there is a large body of evidence on SCFA and MCFA maintaining the desired performance in the absence of antibiotics. In their literature review, Polycarpo et al. [24] report up to $5.67 \%$ improvement in FCR in microbiologically challenged broiler chickens fed these organic acids.

The $\beta-1,4$ mannobiose originating from hydrolyzed copra meal as used in the synergistic blend has been linked to Salmonella reduction in the cecum of broiler chickens challenged with Salmonella at two weeks of age [16]. The same ingredient is shown to improve immune response, by increased IgA levels in feces and upregulated immunerelated genes in the ileum, in non-challenged broiler chickens [25]. In addition, in vitro data show increased phagocytosis by macrophages after exposure to $B-1,4$ mannobiose [15]. In line with the current study, B-1,4 mannobiose did improve final body weight and feed efficiency [26]. In another study, Ibuki et al. [27] showed an improved body weight $(+36.3 \mathrm{~g})$, breast meat $(+12.4 \mathrm{~g})$ and thigh weights $(+9.3 \mathrm{~g})$ at 22 days of age in broiler chickens fed $\mathrm{\beta}-1,4$ mannobiose. In contrast, Ibuki et al. [25] observed no significant effects of this ingredient on growth performance.

Another ingredient in the blend, butyric acid has been described to reduce fecal shedding of Salmonella Enteridis when administered in protected coated form or in the form of impregnated microbeads $[12,28,29]$. Sodium butyrate improves gut health by 
improving the gut wall and mucosa and improving the microbial balance [13]. Under non-challenged conditions, butyric acid supplemented at $0.4 \%$ or $0.6 \%$ improved final body weight and FCR to the same extent or even numerically higher than the antibiotic treatment in comparison with the negative control [14]. The FCR improvement $(0.52 \%)$ by the synergistic blend as observed in the current study is smaller compared to literature describing single ingredients. In line with the current study, the results described by Aljumaah et al. [30], showed a numerically improved FCR in Salmonella Typhimurium infected chickens when fed the synergistic blend, compared to the infected control birds (1.554 vs. 1.646, respectively). It is important to note that the difference in effect on growth performance between the literature and the current study may be related to the type and inclusion level of organic acids used in the synergistic blend, but also to the study designs having the main focus on Salmonella reduction in the current study.

The current synergistic blend did reduce the cecal counts of Salmonella significantly, however it did not completely eliminate Salmonella in the cecum. This is in line with Abudabos et al. [31], who observed a numerical reduction from 4.38 to $1.83 \log$ CFU/g cecal Salmonella (Typhimurium) when birds were fed a blend of organic acids similar to that used in the current study compared to the infected control birds. In contrast to the cecum, Abudabos et al. [31] describe a complete elimination of Salmonella in the ileum when feeding the organic acid blend. It may be argued that complete elimination of Salmonella was not expected, due to the relatively high number of Salmonella in the inoculum used to get a successful model.

\section{Conclusions}

The current study shows that supplementing a synergistic blend containing a mixture of SCFA, MCFA, hydrolyzed copra meal (containing B-1,4 mannobiose) and microencapsulated sodium salts of butyric acid as main ingredients, significantly reduced cecal Salmonella counts within the first 14 days post-inoculation compared to a control diet. The variety in study designs included in the meta-analysis indicates that the synergistic blend is effective under a wide variety of conditions. In addition, the synergistic blend tended to improve FCR over the entire growth period.

Author Contributions: Conceptualization, S.J.A.v.K. and Y.H.; Methodology, S.J.A.v.K.; Formal Analysis, S.J.A.v.K.; Resources, Y.H.; Writing—Original Draft Preparation, S.J.A.v.K.; Writing-Review \& Editing, Y.H.; Supervision, Y.H. All authors have read and agreed to the published version of the manuscript.

Funding: This research received no external funding.

Institutional Review Board Statement: All animal procedures in the different studies were approved by the local animal committees according to the standard procedures of the universities/institutes, where the studies were performed.

Informed Consent Statement: Not applicable.

Data Availability Statement: The data presented in this study are available on request from the corresponding author. The data are not publicly available due to confidentiality between the contributed parties.

Acknowledgments: The authors would like to thank the different researches and research partners who were involved in the execution of the individual trials. Ana Isabel Garcia Ruiz and her team of the Trouw Nutrition Poultry Research Centre in Spain. Enric Esteve, Ignacio Badiola and Joan Tarradas from IRTA, Spain. Karolina Von Zuben Augusto for coordinating the studies done in Brazil in collaboration with Alberto Back at Mercolab and Antonio José Piantino Ferreira at USP. Lane Pineda for coordinating the study done in Thailand. The researchers mentioned above were only involved in the execution of the individual trials. They were not involved in compiling all data and the execution of the meta-analysis. The authors would like to thank Bart D'heer, Ana Belen Cortez Quiroga, Ellen Hambrecht, Maarten van der Heijden and Coen Smits for their input throughout the procedure of the meta-analysis. 
Conflicts of Interest: The authors of the manuscript are employed by Trouw Nutrition, a company with commercial interests in the described synergistic blend. Trouw Nutrition R\&D adheres to the principles of the European Code of Conduct for Research Integrity [32].

\section{References}

1. Castro-Vargas, R.E.; Herrera-Sánchez, M.P.; Rodríguez-Hernández, R.; Rondón-Barragán, I.S. Antibiotic resistance in Salmonella spp. Isolated from poultry: A global overview. Vet. World 2020, 13, 2070-2084. [CrossRef]

2. $\quad$ Eng, S.-K.; Pusparajah, P.; Ab Mutalib, N.-S.; Ser, H.L.; Chan, K.-G.; Lee, L.-H. Salmonella: A review on pathogenesis, epidemiology and antibiotic resistance. Front. Life Sci. 2015, 8, 284-293. [CrossRef]

3. Hoffman, S.; Devleesschauwer, B.; Aspinall, W.; Cooke, R.; Corrigan, T.; Havelaar, A.; Angulo, F.; Gibb, H.; Kirk, M.; Lake, R.; et al. Attribution of global foodborne disease to specific foods: Findings from a World Health Organisation structured expert elicitation. PLoS ONE 2017, 12, e0183641. [CrossRef] [PubMed]

4. Jones, F.T.; Richardson, K.E. Salmonella in commercially manufactured feeds. Poult. Sci. 2004, 83, 384-391. [CrossRef] [PubMed]

5. Van Immerseel, F.; De Zutter, L.; Houf, K.; Pasmans, F.; Haesebrouck, F.; Ducatelle, R. Strategies to control Salmonella in the broiler production chain. World Poult. Sci. J. 2009, 65, 367-392. [CrossRef]

6. Chlebicz, A.; Slizewska, K. Campylobacteriosis, salmonellosis, yersiniosis, and listeriosis as zoonotic foodborne diseases: A review. Int. J. Environ. Res. Public Health 2018, 15, 863. [CrossRef] [PubMed]

7. Nair, D.V.T.; Venkitanarayanan, K.; Johny, A.K. Antibiotic-resistant Salmonella in the food supply and the potential role of antibiotic alternatives for control. Foods 2018, 7, 167. [CrossRef] [PubMed]

8. Pulido-Landinez, M. Food Safety-Salmonella update in broilers. Anim. Feed Sci. Technol. 2019, 250, 53-58. [CrossRef]

9. Van Immerseel, F.; Cauwerts, K.; Devriese, L.A.; Haesebrouck, F.; Ducatelle, R. Feed additives to control Salmonella in poultry. World Poult. Sci. J. 2002, 58, 501-513. [CrossRef]

10. $\mathrm{Li}, \mathrm{L}$. The strategic use of organic acids to improve gut health in poultry. In Proceedings of the 21st Annual Australian Poultry Science Symposium, Sydney, NSW, Australia, 1-3 February 2010; pp. 115-117.

11. Wales, A.D.; Allen, V.M.; Davies, R.H. Chemical treatment of animal feed and water for the control of Salmonella. Foodborne Pathog. Dis. 2010, 7, 3-15. [CrossRef]

12. Van Immerseel, F.; Boyen, F.; Gantois, I.; Timbermont, L.; Bohez, L.; Pasmans, F.; Haesebrouck, F.; Ducatelle, R. Supplementation of coated butyric acid in the feed reduces colonization and shedding of Salmonella in poultry. Poult. Sci. 2005, 84, 1851-1856. [CrossRef]

13. Elnesr, S.S.; Alagawany, M.; Elwan, H.A.M.; Fathi, M.A.; Farag, M.R. Effect of sodium butyrate on intestinal health of poultryReview. Ann. Anim. Sci. 2020, 20, 29-41. [CrossRef]

14. Panda, A.K.; Rama Rao, S.V.; Raju, M.V.L.N.; Shyam Sunder, G. Effect of butyric acid on performance, gastrointestinal tract health and carcass characteristics in broiler chickens. Asian-Australas. J. Anim. Sci. 2009, 22, 1026-1031. [CrossRef]

15. Ibuki, M.; Kovacs-Nolan, J.; Fukui, K.; Kanatani, H.; Mine, Y. ß 1-4 mannobiose enhances Salmonella-killing activity and activates innate immune responses in chicken macrophages. Vet. Immunol. Immunopathol. 2011, 139, 289-295. [CrossRef] [PubMed]

16. Agunos, A.; Ibuki, M.; Yokomizo, F. Effect of dietary B 1-4 mannobiose in the prevention of Salmonella enteritidis infection in broilers. Br. Poult. Sci. 2007, 48, 331-341. [CrossRef] [PubMed]

17. National Research Council. Nutrient Requirements of Poultry, 9th ed.; The National Academies Press: Washington, DC, USA, 1994.

18. Andino, A.; Hanning, I. Salmonella enterica: Survival, colonization, and virulence differences among serovars. Sci. World J. 2015. [CrossRef] [PubMed]

19. Stern, N.J. Salmonella species and Campylobacter jejuni cecal colonization model in broilers. Poult. Sci. 2008, 87, 2399-2403. [CrossRef]

20. EFSA BIOHAZ Panel (EFSA Panel on Biological Hazards); Kousoumanis, K.; Allende, A.; Alvarez-Ordóñez, A.; Bolton, D.; Bover-Cid, S.; Chemaly, M.; De Cesare, A.; Herman, L.; Hilbert, F.; et al. Scientific opinion on the Salmonella control in poultry flocks and its public health impact. EFSA J. 2019, 17, 5596.

21. EFSA and ECDC (European Food Safety Authority and European Centre for Disease Prevention and Control). The European Union One Health 2018 Zoonosis report. EFSA J. 2019, 17, 5926.

22. Wang, X.; Biswas, S.; Paudyal, N.; Pan, H.; Li, X.; Fang, W.; Yue, M. Antibiotic resistance in Salmonella typhimurium isolates recovered from the food chain through national antimicrobial resistance monitoring system between 1996 and 2016. Front. Microbiol. 2019, 10, 985. [CrossRef]

23. Van Immerseel, F.; Russell, J.B.; Flythe, M.D.; Gantois, I.; Timbermont, L.; Pasmans, F.; Haesebrouck, F.; Ducatelle, R. The use of organic acids to combat Salmonella in poultry: A mechanistic explanation of the efficacy. Avian Pathol. 2006, 35, 182-188. [CrossRef] [PubMed]

24. Polycarpo, G.V.; Andretta, I.; Kipper, M.; Cruz-Polycarpo, V.C.; Dadalt, J.C.; Rodrigues, P.H.M.; Albuquerque, R. Meta-analytic study of organic acids as an alternative performance-enhancing feed additive to antibiotics for broiler chickens. Poult. Sci. 2017, 96, 3645-3653. [CrossRef] [PubMed]

25. Ibuki, M.; Kovacs-Nolan, J.; Fukui, K.; Kanatani, H.; Mine, Y. Analysis of gut immune-modulating activity of ß-1,4-mannobiose using microarray and real-time reverse transcription polymerase chain reaction. Poult. Sci. 2010, 89, 1894-1904. [CrossRef] [PubMed] 
26. Ibuki, M.; Fukui, K.; Yamauchi, K. Effect of dietary mannanase-hydrolysed copra meal on growth performance and intestinal histology in broiler chickens. J. Anim. Physiol. Anim. Nutr. 2014, 98, 636-642. [CrossRef] [PubMed]

27. Ibuki, M.; Yoshimoto, Y.; Inui, M.; Fukui, K.; Yonemoto, H.; Saneyasu, T.; Honda, K.; Kamisoyama, H. Dietary mannanasehydrolyzed copra meal improves growth and increases muscle weights in growing broiler chickens. Anim. Sci. J. 2014, 85, 562-568. [CrossRef] [PubMed]

28. Van Immerseel, F.; Fievez, V.; de Buck, J.; Pasmans, F.; Martel, A.; Haesebrouck, F.; Ductatelle, R. Microencapsulated short-chain fatty acids in feed modify colonization and invasion early after infection with Salmonella enteritidis in young chickens. Poult. Sci. 2004, 83, 69-74. [CrossRef]

29. Fernández-Rubio, C.; Ordóñez, C.; Abad-González, J.; Garcia-Gallego, A.; Pilar Honrubia, M.; Jose Mallo, J.; Balaña-Fouce, R. Butyric acid-based feed additives help protect broiler chickens from Salmonella Enteritidis infection. Poult. Sci. 2009, 88, 943-948. [CrossRef]

30. Aljumaah, M.R.; Alkhulaifi, M.M.; Abudabos, A.M.; Alabdullatifb, A.; El-Mubarak, A.H.; Al Suliman, A.R.; Stanley, D. Organic acid blend supplementation increases butyrate and acetate production in Salmonella enterica serovar Typhimurium challenged broilers. PLoS ONE 2020, 15, e0232831. [CrossRef]

31. Abudabos, A.M.; Al-Mufarrey, S.I.; Alyemni, A.H.; Yehia, H.M.; Garelnabi, A.R.; Alotybi, M.N. Effect of using organic acids to substitute antimicrobial growth promoters on broiler chickens performance. J. Food Agric. Environ. 2014, 12, 447-451.

32. Drenth, P.J.D. The European code of conduct for research integrity. In Promoting Research Integrity in a Global Environment; World Scientific: Singapore, 2012. 